\title{
Hypercentral constituent quark model and isospin dependence
}

\author{
M. M. Giannini, E. Santopinto and A. Vassallo \\ June 26, 2021 \\ Dipartimento di Fisica dell'Università di Genova, \\ I.N.F.N., Sezione di Genova \\ via Dodecaneso 33, 16146 Genova, Italy \\ e-mail:giannini@genova.infn.it
}

\begin{abstract}
The constituent quark model based on a hypercentral approach takes into account three-body force effects and standard two-body potential contributions. The quark potential contains a hypercentral interaction, to which a hyperfine term is added. While the hypercentral potential supplies good values for the centroid energies of the resonance multiplets and a realistic set of quark wave functions, the hyperfine splittings are sometimes not sufficient to account for the observed masses. In this work we have introduced an improved form of the hyperfine interaction and an isospin dependent quark potential. The resulting description of the baryon spectrum is very good, also for the Roper resonance, specially thanks to the flavour dependent interaction.
\end{abstract}

PACS numbers: 12.39.Jh,12.39Pn,14.20.Gk

\section{Introduction}

Constituent Quark Models have been recently widely used for the description of the in-

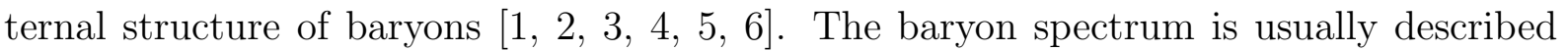
well, although the various models are quite different. However the study of hadron spectroscopy is not sufficient to distinguish among the various forms of quark dynamics. To this end one has to study in a consistent way all the physical observables of interest, in particular, besides the spectrum, the photocouplings, the electromagnetic form factors and the strong decay amplitudes. Such a systematic study of baryon properties is better performed within a general framework, and in this respect a hypercentral approach to 
quark dynamics can be used [6]. The model consists of a hypercentral quark interaction containing a linear plus coulomb-like term, as suggested by lattice QCD calculations [7, 8]. A hyperfine term of the standard form [1] is added and treated as a perturbation. The few free parameters of the model are fitted to the spectrum, the resulting baryon states are then used in order to calculate the various properties of interest, in particular the photocouplings [9], the transition form factors [10, 11] and the elastic electromagnetic form factors of the nucleon [12]. The electromagnetic properties are evaluated using a non relativistic current for pointlike quarks, also taking into account the effects of relativistic corrections [11, 12]. In particular this parameter-free calculation predicts that the ratio of the electric and magnetic proton factors decreases with $Q^{2}$ [13], as shown by the recent TJNAF experiment [14].

The description of the non strange baryon spectrum obtained by the hypercentral Constituent Quark Model (hCQM) [6] is fairly good and comparable to the results of other approaches. In particular, the $\mathrm{SU}(6)$-structure of the levels is accounted for thanks to the spin-independent hypercentral interaction; the $\Delta-N$ mass difference is correctly described by the hyperfine splitting and the theoretical energies of the negative parity resonances are in good agreement with data. However, notwithstanding such overall fair description of the spectrum, in some cases the splittings within the various $\mathrm{SU}(6)$ multiplets are too low. This is particularly true for the Roper resonances and for the higher states. A possible origin of these problems could be the (widespread) use of a $\delta$-like hyperfine interaction. To this end we have introduced different kinds of space smearings; the resulting hyperfine term becomes acceptable from the theoretical point of view, but, as we shall show below, it does not improve the description of the spectrum.

A more important issue is the flavour dependence of the quark interaction. Actually, within the algebraic approach, the quark energy is written in terms of Casimir operators of symmetry groups which are relevant for the three-quark dynamics; in this respect it is quite natural to introduce an isospin dependent term, which turns out to be important for the description of the spectrum [5, 15]. On the other hand, in the chiral constituent quark model recently proposed [4, 16], the splittings are produced by the one-bosonexchange interaction between quarks and therefore a flavour-dependent potential arises, which seems to be important in order to describe the baryon spectrum, at least below $1.7 \mathrm{GeV}$.

In the following, we shall show that in the hCQM a flavour dependent potential can be introduced [17] as a perturbative term leading to improved splittings within the $S U(6)$ multiplets. In particular, in this way, the Roper resonance is reproduced quite well and the higher states acquire a much larger splitting, in agreement with data.

In Section 2 we remind briefly the model and the main results in the description of the spectrum and the electromagnetic excitation of the baryon resonances. In Section 3 we introduce in the hCQM a generalized SU(6)-breaking interaction treated as a perturbation and we show the results of the model compared with the experimental spectrum. Finally, in Section 4 there are some discussions and conclusions. 


\section{The hypercentral model}

The internal quark motion is described by the Jacobi coordinates $\rho$ and $\lambda$ :

$$
\rho=\frac{1}{\sqrt{2}}\left(\mathbf{r}_{1}-\mathbf{r}_{2}\right), \quad \lambda=\frac{1}{\sqrt{6}}\left(\mathbf{r}_{1}+\mathbf{r}_{2}-2 \mathbf{r}_{3}\right)
$$

or equivalently, $\rho, \Omega_{\rho}, \lambda, \Omega_{\lambda}$. In order to describe the three-quark dynamics it is convenient to introduce the hyperspherical coordinates, which are obtained substituting the absolute values $\rho$ and $\lambda$ by

$$
x=\sqrt{\rho^{2}+\lambda^{2}}, \quad \xi=\operatorname{arctg}\left(\frac{\rho}{\lambda}\right),
$$

where $x$ is the hyperradius and $\xi$ the hyperangle. In this way one can use the hyperspherical harmonic formalism [18].

In the hypercentral constituent quark model (hCQM), the quark potential, $V$, is assumed to depend on the hyperradius $x$ only, that is to be hypercentral. Therefore, $V=V(x)$ is in general a three-body potential, since the hyperradius $x$ depends on the coordinates of all the three quarks. Since the potential depends on $x$ only, in the three-quark wave function one can factor out the hyperangular part, which is given by the known hyperspherical harmonics [18]. The remaining hyperradial part of the wave function is determined by the hypercentral Schrödinger equation:

$$
\left[\frac{d^{2}}{d x^{2}}+\frac{5}{x} \frac{d}{d x}-\frac{\gamma(\gamma+4)}{x^{2}}\right] \psi_{[\gamma]}(x)=-2 m[E-V(x)] \psi_{[\gamma]}(x)
$$

where $\psi_{[\gamma]}(x)$ is the hypercentral wave function and $\gamma$ is the grand angular quantum number given by $\gamma=2 v+l_{\rho}+l_{\lambda} ; l_{\rho}$ and $l_{\lambda}$ are the angular momenta associated with the $\rho$ and $\lambda$ variables and $v$ is a non negative integer number.

There are at least two hypercentral potentials which lead to analytical solutions. First, the h.o. potential, which has a two-body character, turns out to be exactly hypercentral, since

$$
\sum_{i<j} \frac{1}{2} k\left(\mathbf{r}_{i}-\mathbf{r}_{j}\right)^{2}=\frac{3}{2} k x^{2}=V_{\text {h.o. }}(x) .
$$

The second one is the 'hypercoulomb' potential [19, 20, 21]

$$
V_{h y c}(x)=-\frac{\tau}{x}
$$

This potential is not confining, however it has interesting properties. In fact it leads to a power-law behaviour of the proton form factor [20] and of all the transition form factors [22, 23]. Moreover it has an exact degeneracy between the first $0^{+}$excited state and the first $1^{-}$states [24, 20, 21], which can be respectively identified with the Roper resonance and the negative parity resonances. This degeneracy seems to be in agreement with phenomenology and is typical of an underlying $\mathrm{O}(7)$ symmetry [21]. This feature cannot be reproduced in models with only two-body forces and/or harmonic oscillator bases since the excited $L=0$ state, having one more node, lies above the $L=1$ state [24]. 
The dynamic symmetry $O(7)$ of the hyperCoulomb problem can be used to obtain the eigenvalues using purely algebraic methods, similarly to what is done in the hydrogen atom case with the $O(4)$ symmetry. In fact, the hyperCoulomb Hamiltonian can be rewritten as 21

$$
H=-\frac{\tau^{2} m}{2\left[C_{2}(O(7))+\frac{25}{4}\right]},
$$

where $C_{2}(O(7))$ is the quadratic Casimir invariant of $O(7)$, and the energy eigenvalues became

$$
E=-\frac{\tau^{2} m}{2(n+5 / 2)^{2}},
$$

where $\mathrm{n}$ is a non negative integer [21].

As a confining hypercentral potential in our model we have assumed a form [6]

$$
V(x)=-\frac{\tau}{x}+\alpha x
$$

that means a coulomb-like term plus a linear confining term as suggested by lattice QCD calculations [7, 8]. In order to describe the splittings within the $S U(6)$-multiplets we introduce a hyperfine interaction of the standard form [1] and we treat it as a perturbation. Having fixed the quark mass $m$ to $1 / 3$ of the nucleon mass, the remaining three free parameters ( $\tau, \alpha$ and the strength of the hyperfine interaction) are fitted to the spectrum. The strength of the hyperfine interaction is determined by the $\Delta$ - Nucleon mass difference and the spectrum is described with $\tau=4.59$ and $\alpha=1.61 \mathrm{fm}^{-2}$. Having fixed the parameters of the potential, the wave functions of the various resonances are completely determined and have been used for the calculation of the photocouplings [9], the transition form factors to the negative parity resonances [10], the elastic form factors [12] and the ratio between the electric and magnetic form factors of the proton 13 .

The resulting overall description of the experimental data is quite good, however there are some problems. As far as the spectrum is concerned, the model fails to reproduce the Roper resonance and the higher levels. The evaluated size of the nucleon is too low, since the resulting r.m.s. radius is $0.46 \mathrm{fm}$ and this affects the description of the elastic form factors. The introduction of relativistic corrections substantially improves the calculated form factors [11, 13] but it is not enough. The strength of the helicity amplitudes are underestimated, as it happens in all constituent quark models.

There may be different reasons for such discrepancies. The splittings within the multiplets are not all adequately described by the hyperfine interaction. One should remind that the form we have assumed for the hyperfine interaction contains a $\delta$-like term, which is troublesome from the theoretical point of view. Furthermore, the splittings can be originated also by other terms, for instance isospin-dependent ones [5, 4].

Another possible reason of the discrepancies is the fact that the model does not contain any explicit quark-pair creation mechanism, which is expected to be particularly important for the description of the eletromagnetic excitation [25, 9, 10] but may lead to some residual effects also in the spectrum [26, 27, 28]. In the latter case, the creation of quark-antiquark pairs could be the microscopic origin of an isospin dependent part of 
the potential. In this respect the constituent quark potential has to be considered as an effective potential in the three-quark subspace, taking into account implicitly the missing Fock-space configurations.

\section{The spin and isospin splittings}

The standard hyperfine interaction is used in order to reproduce the splittings within the $S U(6)$-multiplets. As mentioned above, it contains a $\delta$-like term which is an illegal operator. For this reason we have modified it by introducing a smearing factor given by a gaussian function of the quark pair relative distance [17]:

$$
H_{S}=A_{S} \sum_{i<j} \frac{1}{\left(\sqrt{\pi} \sigma_{S}\right)^{3}} e^{-\frac{r_{i j}^{2}}{\sigma_{S}^{2}}}\left(\mathbf{s}_{i} \cdot \mathbf{s}_{j}\right),
$$

where $\mathbf{s}_{i}$ is the spin operator of the i-th quark and $r_{i j}$ is the relative quark pair coordinate. The results for the spectrum are shown in Fig. 1. The fitted parameters are $\alpha=1.58 \mathrm{fm}^{-2}$, $\tau=4.98, A_{S}=38.4 \mathrm{fm}^{2}, \sigma_{S}=0.8 \mathrm{fm}$. The correct limit for vanishing smearing is obtained, in the sense that the spectrum of Ref. [6] is reproduced.

We have also tried a smearing depending on the hyperradius $x$ only of the form [17]:

$$
H_{\text {Spin }}=B_{S}\left(\frac{1}{\Lambda_{S}} e^{-\frac{x}{\Lambda_{S}}}\right) \sum_{i<j}\left(\mathbf{s}_{i} \cdot \mathbf{s}_{j}\right)
$$

where $\mathbf{s}_{i}$ is the spin operator of the $\mathrm{i}$-th quark and $x$ is the hyperradius. The fitted parameters are $\alpha=1.49 \mathrm{fm}^{-2}, \tau=5.01, B_{S}=196.4 \mathrm{fm}^{2}, \Lambda_{S}=1.6 \mathrm{fm}$. There is an improvement for the higher states, however there is a too strong degeneracy of the levels and the good agreement for the negative parity states obtained in the hCQM [6] is lost. Therefore in the following we shall use only the hyperfine interaction with a two-body smearing.

As quoted in the previous sections, there are different motivations for the introduction of a flavour dependent term in the three-quark interaction. The well known GuerseyRadicati mass formula [15] contains a flavour dependent term, which is essential for the description of the strange baryon spectrum:

$$
\begin{aligned}
\hat{M}=M_{0}+ & a \hat{C}_{2}\left(S U_{S F}(6)\right)+b \hat{C}_{2}\left(S U_{F}(3)\right) \\
& +b^{\prime} \hat{C}_{2}\left(S U_{I}(2)\right)+b^{\prime \prime} \hat{C}_{1}\left(U_{Y}(1)\right) \\
& +b^{\prime \prime \prime} \hat{C}_{2}\left(U_{Y}(1)\right)+\hat{C}_{2}\left(S U_{S}(2)\right),
\end{aligned}
$$

where $M_{0}$ is fixed for any $S U(6)$-multiplet, $\hat{C}_{2}(S U(N))$ is the Casimir of $\mathrm{SU}(\mathrm{N}), Y$ is the hypercharge, $I$ is the total isospin and $S$ the total spin. For non strange baryons this formula implies an isospin dependence. In the algebraic description of baryon properties [5], the space part of the mass operator is written in terms of the generators of the $\mathrm{U}(7)$ group, while for the internal degrees of freedom the Guersey-Radicati mass formula 
[15] is used. In the chiral Constituent Quark Model [4, [16], the non confining part of the potential is provided by the interaction with the Goldstone bosons, giving rise to a spin- and isospin-dependent part, which is crucial for the description of the spectrum for energies lower than $1.7 \mathrm{GeV}$. It has been also pointed out quite recently that an isospin dependence of the quark potential can be obtained by means of quark exchange [29]. More generally, one can expect that the quark-antiquark pair production can lead to an effective quark interaction containing an isospin (or flavour) dependent term. On the other hand, the fact that the constituent quark model does not contain explicitly this mechanism is may be the reason why the low $Q^{2}$-behaviour of the electromagnetic transition form factors is not reproduced [25, 9].

With these motivations in mind, we have introduced isospin dependent terms in the hCQM hamiltonian.

To this end we have added two terms in the three-quark hamiltonian with the hyperfine interaction of Eq.(99). The first one depends on the isospin only and has the form:

$$
H_{\mathrm{I}}=A_{I} \sum_{i<j} \frac{1}{\left(\sqrt{\pi} \sigma_{I}\right)^{3}} e^{-\frac{\mathbf{r}_{i j}^{2}}{\sigma_{I}^{2}}\left(\mathbf{t}_{i} \cdot \mathbf{t}_{j}\right),}
$$

where $\mathbf{t}_{i}$ is the isospin operator of the $\mathrm{i}$-th quark and $r_{i j}$ is the relative quark pair coordinate. The second one is a spin-isospin interaction, given by

$$
H_{\mathrm{SI}}=A_{S I} \sum_{i<j} \frac{1}{\left(\sqrt{\pi} \sigma_{S I}\right)^{3}} e^{-\frac{r_{i j}^{2}}{\sigma_{S I}^{2}}}\left(\mathbf{s}_{i} \cdot \mathbf{s}_{j}\right)\left(\mathbf{t}_{i} \cdot \mathbf{t}_{j}\right),
$$

where $\mathbf{s}_{i}$ and $\mathbf{t}_{i}$ are respectively the spin and isospin operators of the i-th quark and $r_{i j}$ is the relative quark pair coordinate. The complete interaction is then given by

$$
H_{\text {int }}=V(x)+H_{\mathrm{S}}+H_{\mathrm{I}}+H_{\mathrm{SI}} .
$$

The resulting spectrum for the $3^{*}$ - and $4^{*}$ - resonances is shown in Fig.2 and in Table I. The $N-\Delta$ mass difference is no more due only to the hyperfine interaction. In fact, in this model its contribution is only about $35 \%$, the remaining splitting comes from the spin-isospin term, (50\%), and from the isospin one, (15\%).

It should also be noted that the inversion between the Roper and the negative parity resonances is almost entirely due to the spin-isospin interaction, as stated in Ref. [4].

The tensor term coming from the hyperfine interaction has been kept as well, however its contribution to the spectrum is negligible.

In Table II and Table III we list all the remaining states predicted by the model and when possible we give a temptative assignation to one- and two-star states. We see that the number of predicted states is higher than the presently observed ones, that is, as in other models, we have the problem of missing resonances. It is interesting to observe that while the quality of the reproduction of the spectrum for the $3^{*}$ - and $4^{*}$ - resonances is quite the same for the various Constituent Quark Models [1, 2, 5, 4, 16], there are different predictions for the other states, that means for $1^{*}, 2^{*}$ and missing resonances. 
Recently in a three-channel multi-resonance amplitude analysis it has been found evidence for a third low-lying $P_{11}$ state at $1740 \pm 11 \mathrm{MeV}$ [30]. The first two $P_{11}$ states at $1439 \pm 19 \mathrm{MeV}$ and $1729 \pm 16 \mathrm{MeV}$ correspond to the N(1440) and N(1710) of the PDG [31]. In the hCQM the first three $P_{11}$ states are at $1463 \mathrm{MeV}, 1752 \mathrm{MeV}$ and $1828 \mathrm{MeV}$ respectively. A new analysis of kaon photoproduction data [32] has shown evidence for a third $D_{13}$ resonance at $1895 \mathrm{MeV}$, which can be described by one of the states predicted by the present model (see Table II).

\section{Discussions and conclusions}

A considerable improvement in the description of the spectrum is obtained with an isospin dependent potential. As quoted in the previous section, a possible motivation of the isospin-dependent terms of the quark interaction is given by quark-antiquark pair production mechanisms.

This kind of mechanisms have been studied within the string model in connection with the quenching problem of quark models in the meson spectrum [26, 27, 28]. Their contributions turn out to be mainly spin-independent and can be reabsorbed into the string parameter, so the residual part can be considered as a perturbative correction. This can be considered a suggestion also for the baryon case, even if there are differences. Therefore, any interaction leading to unperturbed states with reasonable spin-averaged values of the energy levels can be considered to contain implicitly this kind of contribution. The splittings, which are in general spin and isospin dependent, can be treated perturbatively.

In this article we have shown that the complete interaction including spin and isospin terms (see Eq.(14)), reproduces the position of the two Roper resonances of the nucleon, while keeping the good description of the negative parity resonances. It should be noted that also the higher states are accounted for. The hypercentral potential is a good starting point for the construction of an unperturbed spectrum and leads to realistic quark states, as it is shown also by the reproduction of the e.m. form factors and transition form factors, which are sensitive to the wave functions. The configuration mixing is usually a higher order correction, apart from some special cases as the neutron form factor or the $S_{11}(1650)$ helicity amplitude etc. , for which the $S U(6)$-contribution is vanishing. In this respect, the forthcoming more precise data that will be soon available will supply valuable information concerning the possible $S U(6)$-breaking terms in the quark interaction. Finally, one can observe that the various Constituent Quark Models give different results concerning the $1^{*}$ and $2^{*}$ states and the position and number of the missing resonances . Therefore, the expected new data coming from the TJNAF will be very helpful in order to discriminate among thems.

\section{References}

[1] N. Isgur and G. Karl, Phys. Rev. D18, 4187 (1978); D19, 2653 (1979); D20, 1191 (1979); S. Godfrey and N. Isgur, Phys. Rev. D32, 189 (1985); S. 
[2] S. Capstick and N. Isgur, Phys. Rev. D 34,2809 (1986)

[3] M.M. Giannini, Rep. Prog. Phys. 54, 453 (1991).

[4] L. Ya. Glozman and D.O. Riska, Phys. Rep. C268, 263 (1996).

[5] R. Bijker, F. Iachello and A. Leviatan, Ann. Phys. (N.Y.) 236, 69 ( 1994)

[6] M. Ferraris, M.M. Giannini, M. Pizzo, E. Santopinto and L. Tiator, Phys. Lett. B364, 231 (1995).

[7] M. Campostrini,K.Moriarty and C. Rebbi, Phys.Rev. D36, 3450 (1987); L. Heller, in "Quarks and Nuclear Forces", eds. D.C. Vries and B. Zeitnitz, Springer Tracts in Modern Physics 100, 145 (1982).

[8] Gunnar S. Bali et al., Phys. Rev. D62, 054503 (2000); Gunnar S. Bali, Phys. Rep. 343, 1 (2001).

[9] M. Aiello, M. Ferraris, M.M. Giannini, M. Pizzo and E. Santopinto, Phys. Lett. B387, 215 (1996).

[10] M. Aiello, M. M. Giannini, E. Santopinto, J. Phys. G: Nucl. Part. Phys. 24, 753 (1998)

[11] M. De Sanctis, E. Santopinto, M.M. Giannini, Eur. Phys. J. A2, 403 (1998).

[12] M. De Sanctis, E. Santopinto, M.M. Giannini, Eur. Phys. J. A1, 187 (1998).

[13] M. De Sanctis, M.M. Giannini, L. Repetto, E. Santopinto, Phys. Rev. C62,025208 (2000).

[14] M.K. Jones et al., Phys. Rev. Lett. B84,1398 (2000).

[15] F. Guersey and L.A. Radicati, Phys. Rev. Lett. 13, 173 (1964); M. Gell-Mann, Phys. Rev. 125, 1067 (1962); S. Okubo, Prog. Theor. Phys. 27, 949 (1962)

[16] L. Ya. Glozman, Z. Papp, W. Plessas, K. Varga, R. F. Wagenbrunn, Phys. Rev. C57, 3406 (1998); L. Ya. Glozman, W. Plessas, K. Varga, R. F. Wagenbrunn, Phys. Rev. D58, 094030 (1998).

[17] A. Vassallo, Thesis, Genova 2000 (unpublished)

[18] G. Morpurgo, Nuovo Cimento 9, 461 (1952); Yu. A. Simonov, Sov. J. Nucl. Phys. 3, 461 (1966); J. Ballot and M. Fabre de la Ripelle, Ann. of Phys. (N.Y.) 127, 62 (1980); M. Fabre de la Ripelle, in "Models and Methods in Few-Body Physics" (L.S. Ferreira, A. C. Fonseca and L. Streit eds.) Lecture Notes in Physics 273, Springer (Berlin), 1987, p. 283

[19] M. Fabre de la Ripelle and J. Navarro, Ann. Phys. (N.Y.) 123, 185 (1979). 
[20] H.J. Lipkin, Rivista Nuovo Cimento I (volume speciale), 134 (1969); J. Leal Ferreira and P. Leal Ferreira, Lett. Nuovo Cimento vol. III, 43 (1970); M.M. Giannini, Nuovo Cimento A76, 455 (1983); D. Drechsel, M.M. Giannini and L. Tiator, in "The Three-Body Force in the Three-Nucleon System", eds. B.L. Berman and B.F. Gibson, Lecture Notes in Physics 260, 509 (1986); Few-Body Syst. Suppl. 2, J.-L. Ballot and M. Fabre de la Ripelle eds. , 448 (1987).

[21] E. Santopinto, M.M. Giannini and F. Iachello, in "Symmetries in Science VII", ed. B. Gruber, Plenum Press, New York, 445 (1995); F. Iachello, in "Symmetries in Science VII", ed. B. Gruber, Plenum Press, New York, 213 (1995).

[22] E. Santopinto, F. Iachello and M.M. Giannini, Nucl. Phys. A623, 100c (1997).

[23] E. Santopinto, F. Iachello and M.M. Giannini, Eur. Phys. J. A1, 307 (1998).

[24] J.-M. Richard, Phys. Rep. 212, 1 (1992); H. Hogaasen and J.-M. Richard, Phys. Lett. B 124, 520 (1983).

[25] F. Iachello, Proceedings of the $1^{\text {st }}$ International Conference on Perspectives in Hadronic Physics, ICTP Trieste 12-16 may 1997, (S. Boffi, C. Ciofi degli Atti and M. M. Giannini eds.), World Scientific, Singapore 1998, p. 363.

[26] P. Geiger and N. Isgur, Phys. Rev. D 41, 1595 (1990); Phys. Rev. D 44, 799 (1991); Phys. Rev. Lett. 67, 1066 (1991); Phys. Rev. D 47, 5050 (1993); Phys. Rev. D 55, 299 (1997).

[27] N. Isgur and J. Paton, Phys. Rev. D 312910 (1985).

[28] N. Isgur, Phys. Rev. D 62, 054026 (2000).

[29] G. Dillon and G. Morpurgo, Z. Phys. C73, 547 (1997).

[30] M.Q. Tran et al., Phys. Lett. B445, 20 (1998).

[31] Particle Data Group, Eur. Phys. J. C15, 1 (2000).

[32] T.Mart and C. Bennhold, Phys. Rev. C61, 012201 (2000). 
Figure 1: The spectrum obtained with the hypercentral potential, Eq.(2.8), and the spin dependent term, Eq.(3.1). The fitted parameters are $\alpha=1.58 \mathrm{fm}^{-2}$, $\tau=4.98, A=38.4 \mathrm{fm}^{2}, \sigma=0.8 \mathrm{fm}$.

Figure 2: The spectrum obtained with the complete interaction of Eq.(3.6), that means the hypercentral potential, of Eq.(2.8), plus the spin dependent term, the isospin interaction and the spin-isospin one (Eqs.(3.1),(3.4),(3.5)). The fitted parameters are $\alpha=1.17 \mathrm{fm}^{-2}, \tau=4.95, A_{S}=67.4 \mathrm{fm}^{2}, \sigma_{S}=2.87 \mathrm{fm}, A_{I}=51.7 \mathrm{fm}^{2}, \sigma_{I}=3.45 \mathrm{fm}$, $A_{S I}=-106.2 \mathrm{fm}^{2}, \sigma_{S I}=2.31 \mathrm{fm}$.

Table I: Mass Spectrum of Nonstrange Baryon Resonances.

\begin{tabular}{lcccr} 
Baryon & Status & $\begin{array}{c}M_{\text {exp }} \\
(\mathrm{MeV})\end{array}$ & $J^{\pi}$ & $\begin{array}{r}M_{\text {theor }} \\
(\mathrm{MeV})\end{array}$ \\
\hline $\mathrm{N}(938) P_{11}$ & $* * * *$ & 938 & $\frac{1}{2}^{+}$ & 938 \\
$\Delta(1232) P_{33}$ & $* * * *$ & $1230-1234$ & $\frac{3}{2}^{+}$ & 1232 \\
$\mathrm{~N}(1440) P_{11}$ & $* * * *$ & $1430-1470$ & $\frac{1}{2}^{+}$ & 1463 \\
$\Delta(1600) P_{33}$ & $* * *$ & $1550-1700$ & $\frac{3}{2}^{+}$ & 1727 \\
$\mathrm{~N}(1535) S_{11}$ & $* * * *$ & $1520-1555$ & $\frac{1}{2}^{-}$ & 1524 \\
$\mathrm{~N}(1520) D_{13}$ & $* * * *$ & $1515-1530$ & $\frac{3}{2}^{-}$ & 1524 \\
$\mathrm{~N}(1650) S_{11}$ & $* * * *$ & $1640-1680$ & $\frac{1}{2}^{-}$ & 1688 \\
$\mathrm{~N}(1700) D_{13}$ & $* * *$ & $1650-1750$ & $\frac{3}{2}^{-}$ & 1692 \\
$\mathrm{~N}(1675) D_{15}$ & $* * * *$ & $1670-1785$ & $\frac{5}{2}^{-}$ & 1668 \\
$\Delta(1620) S_{31}$ & $* * * *$ & $1615-1675$ & $\frac{1}{2}^{-}$ & 1573 \\
$\Delta(1700) D_{33}$ & $* * * *$ & $1670-1770$ & $\frac{3}{2}^{-}$ & 1573 \\
$\mathrm{~N}(1710) P_{11}$ & $* * *$ & $1680-1740$ & $\frac{1}{2}^{+}$ & 1752 \\
$\mathrm{~N}(1720) P_{13}$ & $* * * *$ & $1650-1750$ & $\frac{3}{2}^{+}$ & 1648 \\
$\mathrm{~N}(1680) F_{15}$ & $* * * *$ & $1675-1690$ & $\frac{5}{2}^{+}$ & 1680 \\
$\Delta(1910) P_{31}$ & $* * * *$ & $1870-1920$ & $\frac{1}{2}^{+}$ & 1953 \\
$\Delta(1920) P_{33}$ & $* * *$ & $1900-1970$ & $\frac{3}{2}^{+}$ & 1921 \\
$\Delta(1905) F_{35}$ & $* * * *$ & $1870-1920$ & $\frac{5}{2}^{+}$ & 1901 \\
$\Delta(1950) F_{37}$ & $* * * *$ & $1940-1960$ & $\frac{7}{2}^{+}$ & 1955 \\
$\Delta(1900) S_{31}$ & $* * *$ & $1850-1950$ & $\frac{1}{2}^{-}$ & 1910
\end{tabular}


Table II: All calculated Nucleon Resonances (in MeV) below $2 \mathrm{GeV}$. Tentative assignments of 1- and 2- star resonances are shown in brackets.

\begin{tabular}{ccc} 
State & $\mathrm{M}_{\text {theor }}$ & Baryon \\
\hline$N^{\frac{1}{2}^{+}}$ & 938 & $N(938) P_{11}$ \\
$N^{\frac{1}{2}^{+}}$ & 1463 & $N(1440) P_{11}$ \\
$N^{\frac{1}{2}^{+}}$ & 1752 & $N(1710) P_{11}$ \\
$N^{\frac{1}{2}^{+}}$ & 1828 & \\
$N^{\frac{1}{2}^{+}}$ & 1894 & \\
$N^{1^{+}}$ & 1938 & {$\left[N(2100) P_{11}\right]$} \\
$N^{\frac{1}{2}^{-}}$ & 1524 & $N(1535) S_{11}$ \\
$N^{\frac{1}{2}^{-}}$ & 1688 & $N(1650) S_{11}$ \\
$N^{\frac{1}{2}^{-}}$ & 1861 & \\
$N^{\frac{1^{-}}{2}}$ & 2008 & {$\left[N(2090) S_{11}\right]$} \\
$N^{\frac{3}{2}^{-}}$ & 1524 & $N(1520) D_{13}$ \\
$N^{3^{-}}$ & 1692 & $N(1700) D_{13}$ \\
$N^{3^{-}}$ & 1860 & \\
$N^{3^{-}}$ & 2008 & {$\left[N(2080) D_{13}\right]$} \\
$N^{3^{+}}$ & 1648 & $N(1720) P_{13}$ \\
$N^{3^{+}}$ & 1816 & \\
$N^{\frac{3}{2}}$ & 1894 & {$\left[N(1900) P_{13}\right]$} \\
$N^{3^{+}}$ & 1939 & \\
$N^{3^{+}}$ & 2034 & \\
$N^{5^{+}}$ & 1680 & $N(1680) F_{15}$ \\
$N^{5^{+}}$ & 1833 & \\
$N^{5^{+}}$ & 2046 & {$\left[N(2000) F_{15}\right]$} \\
$N^{\frac{7}{2}^{+}}$ & 1939 & {$\left[N(1990) F_{17}\right]$} \\
$N^{\frac{5}{2}}$ & 1668 & $N(1675) D_{15}$ \\
$N^{\frac{5}{2}}$ & 1984 & {$\left[N(2200) D_{15}\right]$}
\end{tabular}


Table III: All calculated Delta Resonances (in MeV) below $2 \mathrm{GeV}$. Tentative assignments of 1- and 2-star resonances are shown in brackets.

\begin{tabular}{|c|c|c|}
\hline State & $\mathrm{M}_{\text {theor }}$ & Baryon \\
\hline$\Delta^{\frac{1}{2}}{ }^{+}$ & 1900 & $\Delta(1910) P_{31}$ \\
\hline$\Delta^{\frac{1}{2}}{ }^{+}$ & 1953 & \\
\hline$\Delta^{\frac{1}{2}^{-}}$ & 1573 & $\Delta(1620) S_{31}$ \\
\hline$\Delta^{\frac{1}{2}^{-}}$ & 1910 & $\Delta(1900) S_{31}$ \\
\hline$\Delta^{\frac{3}{2}}{ }^{+}$ & 1232 & $\Delta(1232) P_{33}$ \\
\hline$\Delta^{\frac{3}{2}}{ }^{+}$ & 1727 & $\Delta(1600) P_{33}$ \\
\hline$\Delta^{\frac{3}{2}}{ }^{+}$ & 1921 & $\Delta(1920) P_{33}$ \\
\hline$\Delta^{\frac{3}{2}}{ }^{+}$ & 1955 & \\
\hline$\Delta^{\frac{3}{2}}{ }^{+}$ & 2049 & \\
\hline$\Delta^{\frac{3}{2}}{ }^{-}$ & 1573 & $\Delta(1700) D_{33}$ \\
\hline$\Delta^{\frac{3}{2}^{-}}$ & 1900 & {$\left[\begin{array}{lll}\Delta(1940) & D_{33}\end{array}\right]$} \\
\hline$\Delta^{\frac{5}{2}}{ }^{+}$ & 1901 & $\Delta(1905) F_{35}$ \\
\hline$\Delta^{\frac{5}{2}^{+}}$ & 1956 & {$\left[\Delta(2000) F_{35}\right]$} \\
\hline$\Delta^{\frac{7^{+}}{2}}$ & 1955 & $\Delta(1950) F_{37}$ \\
\hline
\end{tabular}




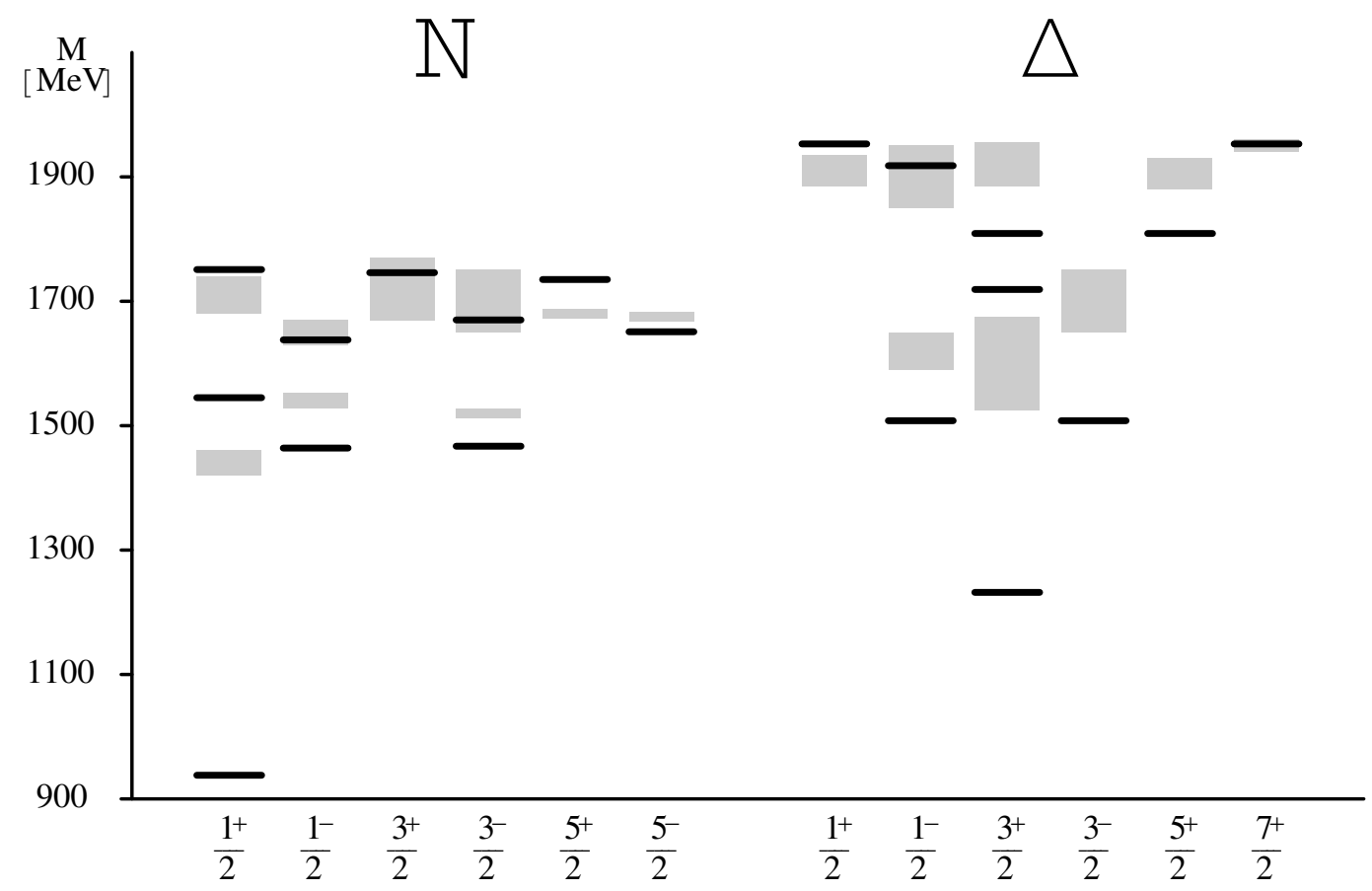




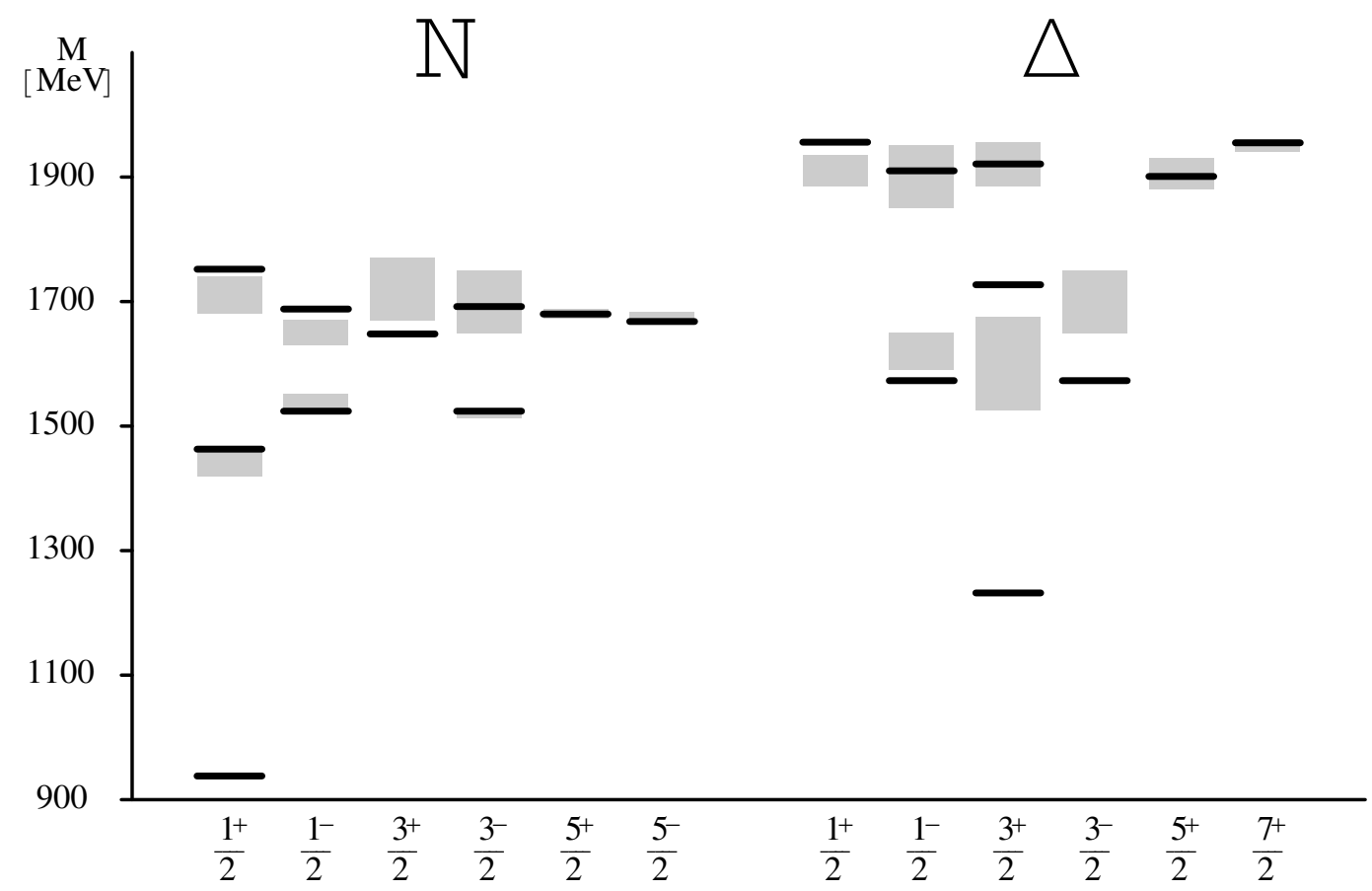

